\title{
Cytokine Storm in Novel Corona Virus Disease (COVID-19)
}

\author{
Mehnaj Begum \\ Type of Article: Review \\ Conflict of Interest: None \\ Funding: DMIMS \\ Ethical Approval: IEC, DMIMS, Wardha
}

Medical Intern, Jawaharlal Nehru Medical College, Datta Meghe Institute of Medical Sciences (Deemed to be University), Sawangi (Meghe), Wardha-442001, Maharashtra, India

\begin{abstract}
Coronavirus disease 2019 (COVID-19) is the latest global issue which has threatened the medical fraternity since its earliest reports on December 31, 2019 in Wuhan, China, and has had a negative impact on global healthcare systems disturbing every aspect of normal human life. COVID-19 infection is distinguished by scope of various epidemiological and pathological features, including high cytokine levels in the blood, leading to an uncontrolled abnormalityresponse known as "cytokine storm".

Accumulating evidence from various clinical trials and studies suggest that this cytokine storm is related with increased intensity of COVID-19 leading to mortality. It is essential to urgently establish the immunopathogenesis of the COVID-19 cytokine storm in the search of proper therapy of COVID disease.In this review article we have discussed the presentcomprehension of the attributes of COVID-19 and the clinical features, immunopathological mechanisms, and management of the COVID-19 induced cytokine storm, especially in severe cases.

Various clinical trials in COVID-19 infected patientshas shown a strong evidence between cytokine production upregulation in severe and critical COVID-19 infected patients and mortality. Considering that the majority of cases of respiratory system and multiorgan failure are caused due to the continuous massive cytokine release, implementation of therapeutics that attenuate this overregulated inflammatory response can perhaps be a possible treatment for severe COVID-19 patients. Thus therapeutic methods for cytokine storm treatment in the pathology of severe COVID-19 infection warrant special attention.

We have discussed the immunopathogenesis of severe COVID-19 infection and the induced cytokine storm and its. In addition, we explored treatment approaches to reverse overactive inflammation in severe COVID-19 infected patients.
\end{abstract}

Keywords: Cytokine storm, Novel Corona virus, Covid-19.

\section{Introduction}

The coronavirus disease (COVID-19) is presentlythe global health concern whichhasdistraught the medical community since itsinitial reports from Wuhan, Hubei province, China on $31^{\text {st }}$ December, 2019. It is said to have been originated from bats, a well-known natural reservoir, and transmitted through unknown intermediary animals to humans. The WHO officially declared the COVID19 as a pandemic on 30 January 2020 after it rapidly spread to the whole world calling it public health emergency of international concern. Transmission is by inhalation of infected droplets and incubation period is around 5.8 days (2-14 days). Supportivetreatment is the mainstay of management. Prevention includeshome isolation, social distancing measures, hang hygiene and face masks use in public places. Presently the sole method advised to hamper the rate of viral transmission globally is by maintaining proper social distancing norms. The spectrum of disease is broadthat ranges between asymptomatic state and mild flu like disease to severe ARDS. Evidence suggest that ARDS may be caused due to "cytokine storm" an unrestrained hyperproduction of inflammatory chemokines leading to a sustained systemic hyperinflammatory response. Chemokines are substances with high chemoattractant activity which helps in the recruitment of immune cells during inflammation.

Till date there are limited treatment options for the management of severe 
COVID-19 infection. Thus, Combination drug therapy consistingof an immunomodulatory drug - to downregulate the cytokine storm - and an antiviral drug that dampens viral load could be beneficial in treatment of severe COVID-19.[1,2]

\section{Immunopathogenesis of COVID 19 associated Cytokine storm:}

The information about pathophysiology of COVID-19 is incompletely understoodandushers an urgency to establish other therapeutic methods based on just pathophysiological assumptions. The structural composition of coronaviruses consists of four structural proteins, these are the spike (S), envelop $(\mathrm{E})$, nucleocapsid $(\mathrm{N})$, and membrane $(\mathrm{M})$ proteins. The $\mathrm{S}$ protein protrudes from the surface of virus and is crucial for host attachment and further penetration. Human pulmonary epithelial cells have abundant angiotensin-converting enzyme (ACE)-2 expressed that can act like receptor for the viral particle to initiate infection by binding to it. [3] The virus subsequently enters these cells and undergoes replication and synthesis of new proteins in the cell cytoplasm. Further via exocytosis the new nucleocapsids are transported to the outsidefrom where these newly formed virusinvadeadjoining epithelial cells whilst also propagating as infective doses for transmission via respiratory droplets. There is only a mild immune response generated during this short disease stage during which individuals are veryinfectious, even though they have a relativelysmallamount of virus circulating in the body. [4,5]

A greater immune response is generated when the viral particle travels deeper to the conducting pathways involving the secretion of CXCL-10 and other interferons from virus-laden cells, manifesting as mild disease presenting as fever, weakness, body ache and nonproductive cough. The bulk of patients often stop progressingforward fromthis stage because the immunitysystem contains the infection here. [3]

However,a fifth of all infected patients are seen toextend further to the lower and smaller respiratory tracts, progressing to ARDS and developing severe symptoms. [6]Here the viral propagation continues after invading in the type 2 alveolar epitheliocytes. These pneumocytes laden with virus particles now secretea variety of cytokines and inflammatory biomolecules into the blood stream. [7]

An aggressive inflammatory response known as "cytokine storm" is seen with the discharge of a humongous quantity of inflammatory cytokine markers in these COVID-19 infected patients. This is because of an excessive hyperactive inflammatory reaction of the infected individual'simmune system to the COVID-19 virus causingTh1 cells to produce and release inflammatory cytokines, likeGM-CSF, IL-6, TNF- $\alpha$, etc. High blood levels of interleukin-6 and TNF- $\alpha$ is characteristic of the cytokine storm in COVID-19. [8,9]

Studies show that the increasing severity of COVID-19 infection is related with raised blood levels of inflammatory mediators like IL-2, IL-1, TNF- $\alpha$, CRP and D-dimers among others. Amongst these, IL-6 levels are significantly related with COVID-19 associated CRS mortality. Hence, IL-6 could act as a target for possible therapeutic modality for severe infection. This could be done in either of two ways, either by IL- 6 antibodies or by chemical modulators that block IL-6 signaling. Two IL-6R antagonists, tocilizumab (TCZ) and sarilumab (SAR) are clinically approved and could have significant action in severe, critical COVID-19 infection. The potential immunopathogenesis and treatmentmodalities for severe COVID-19 are discussed here with prime focus on IL6-signallingmechanism[10].

IL-6 is known to suppress normal T-cells while TNF- $\alpha$ promotesapoptosis of $\mathrm{T}$ - 
cellsthereby contributing to lymphocytopenia, leading to secondary bacterial infection in severe COVID cases. [11]

In addition, due to ACE-2 exhaustion because of virus entry, angiotensin II (AngII) levels and metabolism is disrupted, causing an initial raise in AngII levelswhich increases cytokines release and promotes microvascular dysfunction and a prothrombotic setting. [12]

To direct therapeutic strategies, it is imperative to clarify the mechanisms pertaining the immunopathogenesis in patients with COVID-19.

There are two prospective mechanisms of COVID-19-induced immunopathology:

\section{Depletion and exhaustion of lymphocytes -}

$\mathrm{T}$ cells and macrophages can directly be infected by COVID-19 virus, thus it is hypothesized that ACE2 receptors present on lymphocytes (T-cells) promotes virus entry into lymphocytes. [13]It is also seen that higher levels of cytokines is inversely related with reduced number of $T$ cellsdepicting that increased cytokine level promotedT cells depletion as the disease progressed.[14]Furthermore, lymphatic organ function can be directly hamper by the SARS-CoV-2 particle, further inducing lymphopenia.[15] Lastly, it is also reported that increased blood levels of lactic acid in severe COVID-19 patientscan causeinhibition of lymphocyte proliferation.[16]

\section{Cytokine storm -}

An abrupt drastic rise in pro-inflammatory cytokines levels in circulationresults in what is known as a "Cytokine storm". Various immune cells stream into the site of infection from circulation bringing about damaging effects like diffuse alveolar damage, multiorgan damage, septic shock, etc and ARDS as consequences of the cytokine storm which causes low oxygen saturation levels and is major reason forCOVID-19 mortality. [17]
Upon infection with COVID-19, T4 cells arequickly activated into $\mathrm{T}$ helper(Th) 1 cells that secrete GM-CSF that further induce monocytes with increased IL-6 levels thus accelerating inflammation.[18] Thus, in severe forms, immunomodulatory drugsdisrupting the release of cytokinescan be of benefit whentimed appropriately.

\section{Clinical Features}

The clinical symptoms of COVID-19 infection range between asymptomatic state and mild pulmonary disease to severe ARDS, sepsis and multi organ failure. Fever, dry cough, bodyache, joint pain, headache, fatigue, sore throat, and breathlessness are common clinical features reported in COVID-19 infection.[19]

Reports suggest that SARS-CoV-2 genomes have evolved in different clusters from different parts of the world. According to Forster et al report,globally there may be about three core variations of SARS-CoV-2, namely A, B, and C. Different viral isolates display substantial variations in pathogenicity and viral load. Especially, it would be difficult to create a genotype-phenotype relationship given the various clinical symptoms of patients. [20] According to NHC guidelines, a severe case of COVID-19 is defined as:

1. A resting state respiration rate $\geq 30$ breaths $/ \mathrm{min}$;

2. Oxygen saturation; $\mathrm{SpO}_{2} \leq 93 \%$;

3. Arterial blood oxygen partial pressure $\left(\mathrm{PaO}_{2}\right) /$ $\mathrm{FiO}_{2} \leq 300 \mathrm{mmHg}$.

A critical case of COVID-19 is defined as having at least one of these: shock; mechanical ventilation requirement due to respiratory failure; organ failure complication,requiring intensive care. [21] The preponderance in COVID-19present with mild to moderate symptoms, but in about $15 \%$ patients there is progression to severe pneumonia and in 5\% to critical illness. A heightened rise in pro- 
inflammatory cytokines is associated with this progression of disease.

On radiological findings, Pneumonia progression is observed asmulti-focal ground glass opacity, or patchy/segmental consolidation. In later stages patients presentwith acute respiratory distress syndrome (ARDS), acute failure of respiration, liver damage with raised liver enzymes,kidney failure with raised urea and creatinine levels, and multi-organ failure (Chen et all). [22]

COVID-19 patients with lymphopenia are more vulnerable to microbial infections, leading to disease progression and increased severity.[23]

Elderly, people with comorbid conditions, high BMI, low lymphocyte count, and raisedlevels of transaminase, LDH, ddimer, and sIL-2R are factors associated with increased incidence of severe COVID 19 infection.

Complications of severe COVID 19 induced cytokine storm include ARDS, acute respiratory failure, post-COVID19syndrome (long COVID), acute liver and kidney injury, pulmonary embolism, sepsis, DIC.[24,25]

These adverse outcomes are commonly seen in the older patients and in those having co-morbidities. Among patients admitted in intensive care units, a fatality rate of 5 to $10 \%$ is observed. However, in general the COVID-19 case fatality rate is about $1.4-2.5 \%$.

\section{Management Strategies of COVID-19 infection:}

In most circumstances, the therapy is primarily symptomatic and supportive. Initially, according to the symptoms on diagnosis, the patient is classified into mild, moderate or severe and later management is begun.[26,27]

\section{Mild cases:}

In mild cases, i.e, patients presenting with breathlessness $\left(\mathrm{SpO}_{2} 93 \%\right.$ - 97\%), oxygen is administered by a face mask or nasal cannula. If this fails to maintain $\mathrm{SpO}_{2}$ levels a $40 \%$ venturi mask is used to deliver high fixed oxygen. Management is symptomatic with antipyretics and NSAIDs, oral fluids, and nutrition.[28]

\section{Moderate cases:}

In patients with moderate disease $\left(\mathrm{SpO}_{2} 90 \%-94 \%\right)$ High-flow nasal oxygen (HFNO) therapy is used, where the oxygen flow rate higher and fixed. And is adjustable based on patients response.NIV by CPAP usecan be employed in patients who do not respond within an hour.[29]

Other drugs administered in moderate COVID-19 disease include Hydroxychloroquine, intravenous methylprednisolone, and also anticoagulantLMWH for prophylaxis.[30]Antibiotics are recommended for prevention of secondary bacterial infections.

\section{Severe cases:}

In COVID-19 severe cases and in patients with ARDS, urgent $\mathrm{O} 2$ administration is essential at a rate of $5 \mathrm{~L} / \mathrm{min}$ targeting $\mathrm{SpO}_{2}$ level $\geq 90 \% .{ }^{26} \mathrm{HFNO}$ is preferred in decreasingchances of intubation. If the patient developshypercapnia, haemodynamic instability, multiorgan failure, or worsening of $\mathrm{O} 2$ saturation, endotracheal intubation has to be done.[29,31]

Endotracheal intubation is done after preoxygenation with $100 \%$ O2 for 5 minutes, preferably by rapid sequence intubation. Mechanical ventilation is started with low tidal volumes and low inspiratory pressures. 16-18 hours per day of prone position ventilation is feasible and beneficial in conscious patients of COVID-19- pneumonia by rapidly improving blood oxygenation levels but requires utmost precautionary measure to be performed safely. In these patients higher positive end-expiratory pressure (PEEP) is also recommended. In patients with refractory hypoxemia,if feasible, Extracorporeal membrane oxygenation (ECMO) should be considered.[32] 
Other therapies for COVID-19

Antibiotics -There is little data on antibiotic therapies offered to COVID-19 infected patient. Their significance or their indiscriminate or improper use has to be accounted. Broad-spectrum antibiotics are being used in combination. According to a study Wang et al. clarified that patients were administereddrugslike moxifloxacin, ceftriaxone, and azithromycin for effective prevention ofsecondary bacterial infections such as pulmonary infections in patients with viral pneumonias.[33]

Antiviral drugs - Remdesivir is FDA approved for moderate and severe COVID-19 disease, patientsadmitted and put on $\mathrm{O} 2$ supplementation. Favipiravir, another drug, has shownpositive outcome in mild and moderate cases by rapidly reducing viral loadand hence early symptomatic relief.[34]

Corticosteroids - Glucocorticoids are known to decrease $\mathrm{T}$ cell and macrophage proliferation, activation, differentiation, and survival. Methylprednisolone causes inhibition of transcription and action of many different inflammatory cytokines. According to a meta-analysis, it has been shown that corticosteroids are more likely to increase mortality and delay viral clearance in coronavirus infections. However, despite being commonly used, systemic corticosteroids role in COVID-19 is controversial.[35]

Vitamin $C$ - In patients with sepsis and ARDS, it has been observed that vitamin $C$ is quiet efficient due to its actions on oxidative stress and inflammation. The Shanghai Medical Association's expert consensus indicates that a daily dose iv Vitamin Cdaily will contribute oxygenation index betterment.[36]

Heparin -Apart from the anticoagulant effect, heparin, with its anti-inflammatory properties, has potential benefits in COVID-19 infection. Inflammation and production of thrombin are directly related with the bidirectional relationship theory of immune thrombosis, in which inhibition of thrombin formation by heparin reducesthe inflammation response. Heparin's direct anti-inflammatory effects are because of itscapacity of binding to cytokines, neutrophilic chemotaxis inhibition, and migration of leukocytes. [30]

\section{Potential immunotherapeutic treatmentmodalities for Cytokine Storm associated COVID-19:}

Therefore, evidence supportsthat a correlation exists between COVID-19 induced immunopathogenesis and increased intensityof COVID-19 infection among patients.The cornerstone to save severe COVID-19 patients is prevention and by minimizing the cytokine storm. In COVID-19 patients developing cytokine storm the aim must be to revert hyperinflammation.Management with corticosteroids, immunoglobulin, andanticytokine therapies are considered. However, in the survival of critical COVID-19 these treaments have not shown substantial improvement.

Therapeutic strategies that help inLymphocytes enhancement include, immunomodulators, NK cell therapy, or convalescent plasma therapy; whereasstrategies employed to inhibit inflammation includeMSC-therapy, purification of blood and IL-6 antagonists, among others.[37]

Therapeuticmodalitiestarget

thishyperaceractive cytokine response with anticytokine therapies orimmunomodulators, whilstcarefully balancing appropriate inflammatory level for pathogen clearance.[38]

Enhancing lymphocytes agents:

\begin{tabular}{|l|l|l|}
\hline Therapy strategy & Agent & $\underline{\text { Action }}$ \\
\hline $\begin{array}{l}\text { Natural Killer } \\
\text { cell therapy }\end{array}$ & Natural & Antiviral \\
Killer cell & defense \\
\hline $\begin{array}{l}\text { Immunomodulat } \\
\text { ors }\end{array}$ & $\begin{array}{l}\text { IFN-alpha } \\
\text { 2a,2b, P. P. }\end{array}$ & $\begin{array}{l}\text { Innate } \\
\text { antiviral } \\
\text { aeroginosa, }\end{array}$ \\
\hline
\end{tabular}




\begin{tabular}{|l|l|l|}
\hline & thymosin & \\
\hline $\begin{array}{l}\text { Convalescent } \\
\text { plasma therapy }\end{array}$ & $\begin{array}{l}\text { Convalesce } \\
\text { nt plasma }\end{array}$ & $\begin{array}{l}\text { Antibodi } \\
\text { es inhibit } \\
\text { viremia }\end{array}$ \\
\hline
\end{tabular}

Agents that inhibit inflammation: therapycan decrease mortality in critical patients, by raising antibody titers, causing SARS-CoV-2 RNA to disappear, and bringing about significant symptom relief. Cheng et al.[43]reported that reliability ofstarting convalescent plasma therapyin the earlier phase of viremia as its major

\begin{tabular}{|l|l|}
\hline$\underline{\text { Therapeutic strategy }}$ & $\underline{\text { Agent }}$ \\
\hline Purification of blood & Blood purificatio \\
\hline IL-6 signal blockade & Tocilizumab \\
& Sarilumab \\
\hline
\end{tabular}

NK cell immunotherapy -

NK-cell are demonstrated by various trials to mount antigen independent immune response against cancerous cells by their anti-tumour effects.In China NK cell immunotherapy is approved and used for the treatment of COVID-19 by improving immunity.[39]

\section{Immunomodulators -}

In patients infected with SARS-CoV-2, pegylated IFN alfa,has been triedto induce an intrinsic response against the virus. Owing to their immune regulatory roles, treatment modalitieslike P. aeroginosa and thymosin, could prove useful for therapy of COVID-19 patients.[40]

\section{Convalescent Plasma-}

The collection and transfusion of antibodyrich plasma from recovered COVID-19 patients to receptive COVID-19 patients in order to confer them immediate immunity comprises of Convalescent plasma therapy (CPT).The neutralizing antibodies present in a healthy donor's plasma limit virus multiplication and confers immunomodulation by the action of antiinflammatory markers and antibodies.[41] Many trials have observed low rates of mortality among patients undergoing treatment with plasma as compared to patients put on placebo. In another study, Rajendran et al.[42] demonstrated that this action

is direct virus neutralization. Prawbacks like avaitability of plasma, infrastructure, administering timeand consent may restrictusage.

Bargets IL-6R pathways

Blood Purification Systems-

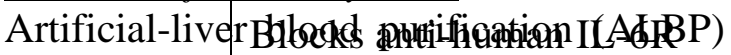
is a procedune that helps to cure illnesses by eliminating toxins and waste from the body. Owing to its success in preventing cytokine storms and suppressing inflammation, blood purification has been approved for the management of cytokine storm in severe infection of COVID-19 in China. It is indicated in fivefold increase or higher level of proinflammatory cytokines or an increase of more than one fold per day, $>10 \%$ lung involvement on Though this treatment modalityis promising cytokine storm management, the proof of itsusefulness in severe infection patients is limited.[44]

\section{IL-6 Inhibitors:}

In the induction of cytokine storm, IL-6 is an important factor as it pilots the cytokine release syndrome. Thus, IL-6 antagonists that target the IL-6 signaling pathwaysor IL-6 receptor (IL-6R) is an encouraging strategy in this regard.

An IgG1 monoclonal antibody, Tocilizumab, acts as antagonist of IL-6 receptor and has been widely used for the management of autoimmune diseases like rheumatoid arthritis.[45]

Clinical trials and studies have demonstrated that tocilizumab, a competitive inhibitor of IL-6 receptorsignalling pathway, is capable of reversing cytokine response syndrome in the T-cell chimeric antigen receptor setting of cancer therapy. Administering imaging, and having comorbidities. 
tocilizumab in the earlier stages of disease may be beneficial in the course of COVID19[46]. $\mathrm{Xu}$ et al.[47] confirmed that no apparent adverse reactions occurred after treatment with tocilizumab, with the remainder recovering well

The effectiveness of sarilumab, IL-6 receptor inhibitor, is assessed in severe and critical cases.Tocilizumab isbeing incorporated in COVID-19 treatment instructions, especially in the management of critical cases with refractory hypoxemia.

\section{JAK Inhibitors -}

Baricitinibis a JAK inhibitor and AAK1 inhibitor, a key regulator in endocytosis of virus that disrupts the entry of the virus into tissuescan be used in treating COVID19 infection. Baricitinib trial use in mild and moderate COVID-19 symptomatic patients is in progress.[48]

\section{Mesenchymal Stem Cells-}

MSC is an immunomodulator known to secrete antimicrobial and painkiller molecules.For the recovery of lung injury due to COVID-19 the use of MSC has been suggested and a clinical trial by Leng et al. demonstrated that administration MSCby iv transplantation is safe and efficaciousin the management of critical severe COVID-19 infection[49,50]. Interesting studies related to immune responses in coronavirus infection were reported [51-54].

\section{Conclusions:}

This new pandemic of 2020 has nevertheless challenged the social, economic, health system and infrastructure globally. In the quest for appropriate therapeutic strategies for COVID-19,many studies, reports and evidence depicts immune system hindrance as the cause of inappropriate CRS and accompanying cytokine storm in the immunopathogenesis of severe and critical COVID-19. While results with certain immunomodulators are promising, clarification about the efficiency, safety, and limits of the different treatments can only be brought after the results of ongoing clinical trials justify so. Tocilizumab is a fine alternative to inhibit the IL-6signalling, a hyperinflammatory pathway mediated by IL-6. Thus a comprehensive approach with the use of antiviral drugs, standard supportive care and imunomodulatory drugs is imperative for care of severe and critical COVID-19 patients.

\section{References:-}

[1] Xinjuan Sun et al, Cytokine storm intervention in the early stages of COVID-19 pneumonia, Cytokine \& Growth Factor Reviews, Volume 53, June 2020, 38-42, ISSN 1359-6101, https://doi.org/10.1016/j.cytogfr.202 $\underline{0.04 .002}$.

[2] $\mathrm{Hu}$ B. et al: The cytokine storm and COVID-19, Journal of Medical Virology, 10.1002/jmv.26396, 0, 0, (2020)

[3] Parasher. ACOVID-19: Current understanding of its pathophysiology, clinical presentation and treatment, Postgraduate Medical Journal Published Online First: 25 September 2020. doi: 10.1136/postgradmedj-2020-138577

[4] Yang, L., Liu, S., Liu, J. et al. COVID-19: immunopathogenesis and Immunotherapeutics. Sig Transduct Target Ther 5, 128 (2020). https://doi.org/10.1038/s41392-02000243-2

[5] Letko M, Marzi A, Munster V, Functional assessment of cell entry and receptor usage for SARS-CoV-2 and other lineage $B$ beta coronaviruses. Nat Microbiol 2020;5:562-9. doi: 10.1038/s41564020-0688-y

[6] $\mathrm{Xu} \mathrm{Z,} \mathrm{Shi} \mathrm{L,} \mathrm{Wang} \mathrm{Y,} \mathrm{et} \mathrm{al.}$ Pathological findings of COVID-19 associated with acute respiratory distress syndrome. Lancet Respir 
Med 2020;8:420-2. doi: 10.1016/S2213-2600(20)30076-X

[7] Tang NL, Chan PK, Wong CK, et al. Early enhanced expression of interferon-inducible protein-10 (CXCL-10) and other chemokines predicts adverse outcome in severe acute respiratory syndrome. Clin Chem 2005;51:2333-40. doi: 10.1373/clinchem.2005.054460

[8] Ragab Dina, Salah H. The COVID19 Cytokine Storm; What We Know So Far. Frontiers in Immunology. Vol. 11. pg1446, June 2020,ISSN 1664-3224

DOI10.3389/fimmu.2020.01446,ww w.frontiersin.org/article/10.3389/fim mu.2020.01446

[9] Haiming W, Xiaoling X, Yonggang $Z$, et al. Aberrant pathogenic GM-CSF+ T cells and inflammatory CD14+CD16+ monocytes in severe pulmonary syndrome patients of a new coronavirus. BioRXiv. https://doi.org/10.1101/2020.02.12.9 $\underline{45576}$

[10] IannacconeG.a - Scacciavillani R. Weathering the Cytokine Storm in COVID-19: Therapeutic Implications. Karger. 2020, Vol.10, No. 5. September 2020, https://doi.org/10.1159/000509483

[11] Huang Q, Wu X, Zheng X, Luo S, $\mathrm{Xu} \mathrm{S}$, Weng J. Targeting inflammation and cytokine storm in COVID-19. Pharmacol Res. 2020;159:105051. doi:10.1016/j.phrs.2020.105051

[12] Perlman, S. \& Dandekar, A. A. Immunopathogenesis of coronavirus infections: implications for SARS. Nat. Rev. Immunol.5, 917-927 (2005).

[13] Thevarajan, I. et al. Breadth of concomitant immune responses prior to patient recovery: a case report of non-severe COVID-19. Nat. Med.26, 453-455 (2020).
[14] Moon, C. Fighting COVID-19 exhausts $\mathrm{T}$ cells. Nat. Rev. Immunol.20, 277 (2020).

[15] Tan, L. et al. Lymphopenia predicts disease severity of COVID-19: a descriptive and predictive study. Signal Transduct. Target Ther.5, 33 (2020).

[16] Yiming L, Kuo S, Early Warning Indicators of Severe COVID-19: A Single-Center Study of Cases From Shanghai, China. Frontiers in Medicine. Vol. 7, 2020. 432. https://www.frontiersin.org/article/10 .3389/fmed.2020.00432

DOI=10.3389/fmed.2020.00432, ISSN=2296-858X

[17] Chen, H. et al. Management of cytokine release syndrome related to CAR-T cell therapy. Front Med.13, 610-617 (2019)

[18] Dholaria BR, Bachmeier CA, Locke F. Mechanisms and Management of Chimeric Antigen Receptor T-Cell Therapy-Related Toxicities. BioDrugs. 2019 Feb;33(1):45-60.

[19] Chen G, Wu D, Guo W, Cao Y, Huang D, Wang $\mathrm{H}$, et al. . Clinical and immunological features of severe and moderate coronavirus disease 2019. J

Clin

Invest. (2020) 130:2620-9. $10.1101 / 2020.02 .16 .20023903$

[20] Forster P., Forster L., Renfrew C., Forster M., Phylogenetic network analysis of SARS-CoV-2 genomes. Proc. Natl. Acad. Sci. U.S.A. 117, 9241-9243 (2020).

[21] National Health Commission of the People's Republic of China Diagnosis and Treatment Protocol for COVID-19 Trial Version 7. (2020). Available online at: http://www.nhc.gov.cn/xcs/zheng cwj/202003/46c9294a7dfe4cef80dc7 f5912eb1989.shtml

[22] Chen N, Zhou M, Dong X, et al. Epidemiological and clinical characteristics of 99 cases of 2019 novel coronavirus pneumonia in 
Wuhan, China: a descriptive study. Lancet. 2020;395(10223):507-513.

[23] Yang, L. et al. Immune characteristics predict outcome of severe and critical COVID-19 patients. Signal Transduct. Target. Ther. in press, (2020).

[24] Xu, Z. et al. Pathological findings of COVID-19 associated with acute respiratory distress syndrome. Lancet Respir. Med.8, 420-422 (2020).

[25] Lippi, G. \&Plebani, M. Laboratory abnormalities in patients with COVID-2019 infection. Clin. Chem Lab. Med. 58, 1131-1134 (2020).

[26] https://www.mohfw.gov.in/pdf/Guid elinesonClinicalManagementofCOVI D1912020.pdf

[27] Cascella M, Rajnik M, Cuomo A, et al., Features, evaluation and treatment coronavirus (COVID-19). Stat pearls [internet]. Treasure Island (FL): Stat Pearls Publishing, Jan 2020.

[28] Whittle, Jessica \& Pavlov, Ivan \&Sacchetti, Alfred \& Atwood, Charles \& Rosenberg, Mark. (2020). Respiratory Support for Adult Patients with COVID-19. Journal of the American College of Emergency Physicians Open. 10.1002/emp2.12071.

[29] Wu CN, Xia LZ, Li KH, et al. Highflow nasal-oxygenation-assisted fibreoptic tracheal intubation in critically ill patients with COVID-19 pneumonia: a prospective randomised controlled trial [2020 Mar 19]. $\mathrm{Br} \quad \mathrm{J}$ Anaesth 2020;125:e166-e168. doi: 10.1016/j.bja.2020.02.020

[30] Kollias A, Kyriakoulis KG, Dimakakos E et al. Thromboembolic risk and anticoagulant therapy in COVID-19 patients: emerging evidence and call for action. $\mathrm{Br} \mathrm{J}$ Haematol 2020;189:846-7. doi: $10.1111 /$ bjh. 16727

[31] World Health Organization . (2020). Clinical management of severe acute respiratory infection (SARI) when COVID-19 disease is suspected: interim guidance, 13 March 2020. World Health Organization.

[32] Prayag S. Respiratory Care for Severe COVID-19. Indian J Crit Care Med 2020;24(7):493-495.

[33] Carla M, Silva V. Implications of antibiotics use during the COVID-19 pandemic: present and future, Journal of Antimicrobial Chemotherapy, Vol 75, Issue 12, December 2020, pages 3413-3416, https://doi.org/10/1093/jac/dkaa350

[34] Beigel JH, Tomashek KM, Dodd LE , et al. Remdesivir for the treatment of COVID-19 - preliminary report [published online ahead of print, 2020 may 22]. N Engl J Med 2020;NEJMoa2007764. doi: 10.1056/NEJMoa2007764

[35] Lee N, Allen Chan KC, Hui DS. et al. Effects of early corticosteroid treatment on plasma SARSassociated Coronavirus RNA concentrations in adult patients. J ClinVirol 2004;31:304-9. doi: 10.1016/j.jcv.2004.07.006

[36] Cheng RZ, Kogan M, Davis D. Ascorbate as Prophylaxis and Therapy for COVID-19-Update From Shanghai and U.S. Medical Institutions. Global Advances in Health and Medicine. January 2020. doi:10.1177/2164956120934768

[37] Yang, L., Liu, S., Liu, J. et al. COVID-19: immunopathogenesis and Immunotherapeutics. Sig Transduct Target Ther 5, 128 (2020). https://doi.org/10.1038/s41392-02000243-2

[38] Tan, M. et al. Immunopathological characteristics of coronavirus disease 2019 cases in Guangzhou, China. Immunology160, 261-268 (2020).

[39] Fang, F., Xiao, W. \& Tian, Z. NK cell-based immunotherapy for cancer. Semin Immunol.31, 37-54 (2017). 
[40] Li, G. \&Clercq, E. D. E. Therapeutic options for the 2019 novel coronavirus (2019-nCoV). Nat. Rev. Drug Discov.19, 149-150 (2020).

[41] Chen, L., Xiong, J., Bao, L. \& Shi, Y. Convalescent plasma as a potential therapy for COVID19. Lancet Infect. Dis.20, 398-400 (2020).

[42] Rajendran K, Krishnasamy N, Rangarajan J, Rathinam J, Natarajan M, Ramachandran A. Convalescent plasma transfusion for the treatment of COVID-19: systematic review. J Med Virol. 2020 May:jmv.25961.

[43] Chen, L., Xiong, J., Bao, L. \& Shi, Y. Convalescent plasma as a potential therapy for COVID19. Lancet Infect. Dis.20, 398-400 (2020).

[44] TuğçeNurYiğenoğlu, TurgayUlas, Extracorporeal blood purification treatment options for COVID-19: The role of immunoadsorption, Transfusion and Apheresis Science, Volume 59, Issue 4,2020,102855, https://doi.org/10.1016/j.transci.2020 .102855 .

[45] Guaraldi G, Meschiari M, CozziLepriA et al. Tocilizumab in patients with severe COVID-19: a retrospective cohort study. Published June 24 2020. doi: 10.1016/S26659913(20)30173-9

[46] Buonaguro FM, Puzanov I, Ascierto PA. Anti-IL6R role in treatment of COVID-19-related ARDS. J Transl Med 2020;18:16. doi: 10.1186/s12967-020-02333-9

[47] $\mathrm{Xu}, \mathrm{X}$. et al. Effective treatment of severe COVID-19 patients with tocilizumab. Proc. Natl Acad. Sci. USA.117, 10970-10975 (2020).

[48] Cantini F, Niccoli L, Matarrese D, Nicastri E, Stobbione P, Goletti D. Baricitinib therapy in COVID-19: A pilot study on safety and clinical impact. J Infect. 2020;81(2):318-356. doi:10.1016/j.jinf.2020.04.017
[49] Leng Z, Zhu R, Transplantation of ACE2- Mesenchymal Stem Cells Improves the Outcome of Patients with COVID-19 Pneumonia. Aging Dis. 2020 Mar 9;11(2):216-228. doi: 10.14336/AD.2020.0228.

[50] $\mathrm{Xu}, \mathrm{X}$. et al. Effective treatment of severe COVID-19 patients with tocilizumab. Proc. Natl Acad. Sci. USA.117, 10970-10975 (2020).

[51] Hande, A., A. Agrawal, A. Sonone, A. Gadbail, M. Gawande, and S. Patil. "Cucurbitacin: As a Candidate against Cytokine Storm in Severe COVID-19 Infection." International Journal of Research in Pharmaceutical Sciences 11, no. Special Issue 1 (2020): 928-30. https://doi.org/10.26452/ijrps.v11iSP L1.3164.

[52] Bawiskar, N., A. Andhale, S. Acharya, S. Kumar, and S. Shukla. "Haematological Manifestations of COVID 19 and Their Prognostic Significance-a Cross-Sectional Study." International Journal of Research in Pharmaceutical Sciences 11, no. Special Issue 1 (2020): 91822.

https://doi.org/10.26452/ijrps.v11iSP L1.3131.

[53] Boharupi, G., and P. Shelotkar. "Immunomodulatory Measures to Strengthen the Body during Covid Outbreak." International Journal of Research in Pharmaceutical Sciences 11, no. Special Issue 1 (2020): 77478 . https://doi.org/10.26452/ijrps.v11iSP L1.3081.

[54] Bhokardankar, P., B. Rathi, M. Khan, and R. Rathi. "COVID-19 Pandemic: Home Remedies for Immunity Boosting." International Journal of Research in Pharmaceutical Sciences 11, no. Special Issue 1 (2020): 734-38. https://doi.org/10.26452/ijrps.v11iSP L1.3075. 